\title{
The study of tilt effect on the preloaded oil pad of hydrostatic turntable
}

\author{
ZhifengLiu ${ }^{1}$,Xiaoyan $\mathrm{Li}^{1}{ }^{1}$ Chengpeng Zhan ${ }^{1}$ \&Yongsheng Zhao ${ }^{1}$ \\ ${ }^{1}$ College of Mechanical Engineering and Applied Electronics Technology,Beijing University of \\ Technology,Beijing 100124, China
}

Keys: hydrostatic turntables; Reynolds equation; thermal fluid; the finite difference method; hydrostatic lubrication

\begin{abstract}
A new method is proposed to analyze thebearing properties ofthe preloaded oil pad in different compensation method when the hydrostatic turntable tilts.Energy equationandReynolds equationare coupled and solved in this paper.Results show that the bearing properties of the preloaded oil pad will be significantly reduced when titling. Andload carrying capacity even decreases by $42 \%$ at maximum tilt angle. All the heat produced in the lubricant film by friction also has a significant impact on the bearingproperties of the preloaded oil pad.
\end{abstract}

\section{Introduction}

Hydrostatic turntables are well known for their high load-carrying capacity, low friction and negligible wear, so they are widely used to carry machine and the large workpiece in the heavy-duty machine tool. Furthermore, numeroushave paid attention to the various aspects of the characteristics of hydrostatic turntable or bearing ${ }^{[1-13]}$.According to references review, we found the investigation of tilt effect is not sufficient.

The preloaded oil pad is an important part of the supporting systemof hydrostatic turntable. In the design of thepad, it is generally assumedthatthe pad surface parallels to the guide surface.However, due to the impact of partial load, manufacturing errors and local deformation, it cannot be avoided that the turntable tilts during being used. Heat is an important factor that can affect the bearing properties of the hydrostatic turntable system. And the increase of the oil temperature not only makes the load carrying capacity lower, but also leads to the thermal deformation ofthe turntable. A new method is proposed to analyze thebearing properties ofthe preloaded oil pad in different compensation method when the hydrostatic turntable tilts. Energy equationandReynolds equationare coupled and solved in this paper.

\section{Theoretical derivation}

\section{Reynolds equation}

In this study, it isassumed that thin film lubrication theory is applicable. So the Reynolds equations can be written as:

We have the simplified energy equations as follows:

$$
\frac{\partial}{\partial r}\left(\frac{r \rho h_{T}^{3}}{12 \eta} \frac{\partial p}{\partial r}\right)+\frac{1}{r} \frac{\partial}{\partial \varphi}\left(\frac{\rho h_{T}^{3}}{12 \eta} \frac{\partial p}{\partial \varphi}\right)=r \rho v_{z}(1)
$$

$$
\begin{gathered}
\rho c\left(u_{r} \frac{\partial T}{\partial r}+\frac{u_{\varphi}}{r} \frac{\partial T}{\partial \varphi}\right)=K \frac{\partial^{2} T}{\partial z^{2}}+\eta\left[\left(\frac{\partial u_{r}}{\partial z}\right)^{2}+\left(\frac{\partial u_{\varphi}}{\partial z}\right)^{2}\right] \\
\eta=\eta_{0} e^{-\beta\left(T-T_{0}\right)} \\
\rho=\rho_{0}\left(1-\lambda\left(T-T_{0}\right)\right)
\end{gathered}
$$




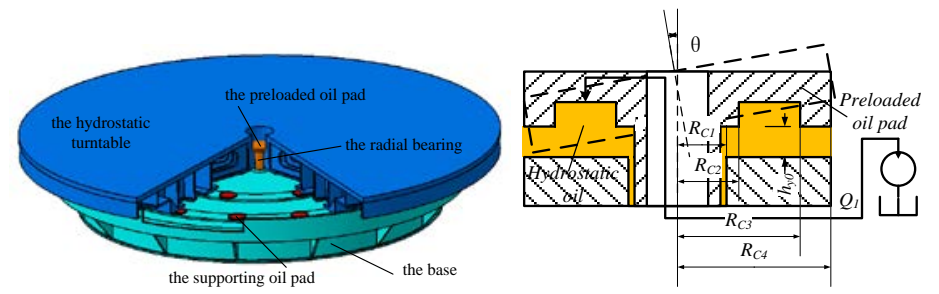

Fig.1.the main structure of the hydrostatic turntable. 2.the preloaded oil pad

\section{Hydrostatic preloaded oil pad}

The main structure of the hydrostatic turntable system is shown in Fig.1. The table and the base are its main structural parts. Meanwhile,the supporting oil pad, the preloaded oil padand the radial bearing is the main component of the turntable support system. The form of the preloaded oil pad is generally annular oil pad, as shown inFig.2. $R_{C 1}, R_{C 2}, R_{C 3}, R_{C 4}$ are the structural parameters of the pad. $h_{y 0}$ is the theoretical film thickness. $Q_{1}$ is flow rate of the pad. $\theta_{\text {isthe tilt angle. }}$

When the preloaded oil pad tilts, the film thickness distribution can be calculated as:

$$
h=h_{y}+r \tan (\theta) \cos (\varphi)
$$

So the recess pressure of the preloaded oil pad can be calculated as:

$$
p_{y 0}=\frac{Q_{1}}{\frac{h_{y 0}^{3}}{\eta_{0}}\left(\int_{0}^{2 \pi}\left(\frac{-\bar{R} \overline{H^{3}}}{12 \overline{\eta_{a}}} \frac{\partial \bar{p}}{\partial \bar{R}}\right)_{\bar{R}=1} d \varphi-\int_{0}^{2 \pi}\left(\frac{-\bar{R} \overline{H^{3}}}{12 \overline{\eta_{a}}} \frac{\partial \bar{p}}{\partial \bar{R}}\right)_{\bar{R}=\frac{R_{C 1}}{R_{C 4}}} d \varphi\right)}
$$

When the pad uses a constant-pressure oil pump for supply and the capillary restrictor for compensation, therecess pressure of hydrostatic oil pad can be calculated as:

$$
p_{y 0}=p_{s}-\frac{128 \eta_{a} l_{c} Q_{1}}{\pi d_{c}^{4}}
$$

As for thehydrostatic oil pad using the orifice restrictor, the recess pressure of hydrostatic oil pad can be calculated as:

$$
p_{y 0}=p_{s}-\frac{\rho}{2}\left(\frac{4 Q_{1}}{\pi a d_{o}^{2}}\right)^{2}
$$
pad.

By integrating oil film pressure distribution,we can obtain the load carrying capacity of the oil

$$
w f_{y}=R_{\mathrm{C} 4}^{2} p_{y 0} \int_{0}^{2 \pi} \int_{R_{\mathrm{C} 1} / R_{\mathrm{C} 4}}^{1} \bar{p} \bar{R} d \bar{R} d \varphi
$$

The load carrying capacity respectively derivation of the film thickness $\bar{H}$ and the axial velocity of guide rail surface $\bar{v}_{z}$, and we can get the stiffness coefficients and the damping coefficients of the oil pad.

$$
\begin{gathered}
K_{\mathrm{z}}=\frac{\partial w f}{h_{0} \partial \bar{H}} \\
\mathrm{C}=\frac{\partial w f}{h_{0} \partial \overline{v_{z}}}
\end{gathered}
$$

The main design parameters of the oil pad and the restrictor are shown in Tab.1. 
Tab.1

\begin{tabular}{|cc|c|c|c|}
\hline \multicolumn{2}{|c|}{ Parameter } & Value & Parameter & Value \\
\hline$R_{C 1}$ & 175 & $R_{C 2} \quad(\mathrm{~mm})$ & 220 \\
\hline$R_{C 3}$ & $(\mathrm{~mm})$ & 235 & $R_{C 4}(\mathrm{~mm})$ & 290 \\
\hline$h_{0}$ & $(\mathrm{~mm})$ & 0.1 & $Q_{1}\left(\mathrm{~m}^{3} / \mathrm{s}\right)$ & $1.73 \mathrm{e}-4$ \\
\hline$p_{s}$ & $(\mathrm{Mpa})$ & 6 & $l_{c}(\mathrm{~mm})$ & 80 \\
\hline \multicolumn{2}{|c|}{$d c(\mathrm{~mm})$} & 2 & $a$ & 0.65 \\
\hline \multicolumn{2}{|c|}{$d o(\mathrm{~mm})$} & 2 & $\eta_{0} \quad(\mathrm{pa} . \mathrm{s})$ & 0.091 \\
\hline$\rho$ & 872 & \multicolumn{2}{|c}{} \\
\hline
\end{tabular}

\section{Result and discussion}

In this paper, when the flow supply means is respectively constant flow supply, capillary restrictor and orifice restrictor, various bearing properties of hydrostatic annular preloaded oil pads with tilt and thermal effects have been calculated. In order to analyze the results, some parameters like: load carrying capacity, recess pressure, stiffness and damping coefficients have been normalized by divide these parameters with no tilting respectively or other parameters.

\section{Recess pressure}

Fig. 3 shows the recess pressure curves at different tilt angles. As shown in the figure, the recess pressure decreases with increasing tilt angle, and initially recess pressure will be slightly decreased when the dimensionless tilt angle $\theta^{*}<0.3$, then recess pressure of the pad will be almost linearly decreased. At the same time, we found that thevariation law of recess pressure with tilt angleswill be changed when the flow supply means is different. First of all, using constant flow supply, the recess pressure is reduced by $44 \%$ at maximum tilt angle. Then, using orifice restrictor, itis reduced by $36 \%$ at maximum tilt angle. At last, using capillary restrictor, itis reduced by $29 \%$ at maximum tilt angle.
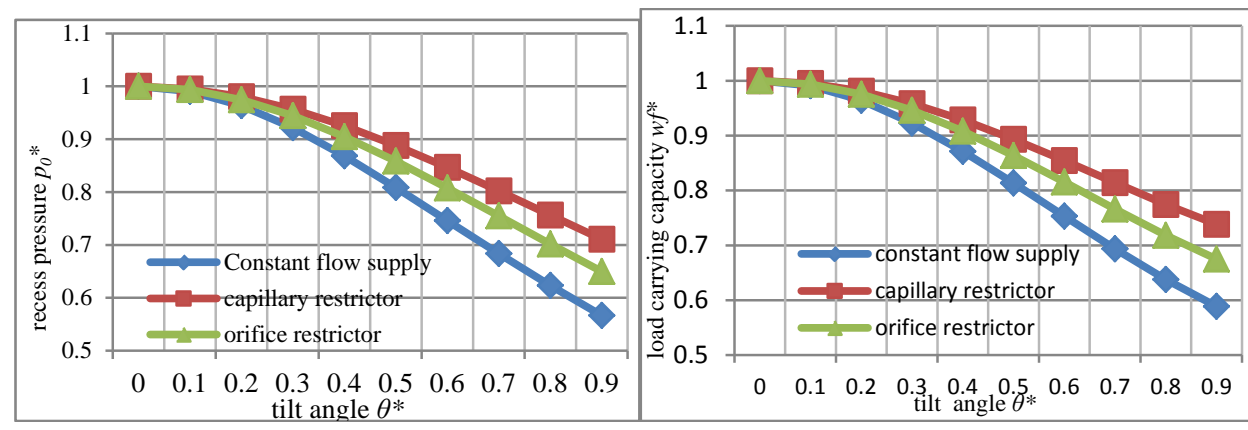

Fig.3 the recess pressure curves Fig.4 the load carrying capacity curves

\section{Load carrying capacity}

The load carrying capacity curvesat different tilt anglesare shown inFig.4. As shown in the figure, the load carrying capacity decreases with increasing tilt angle, similar to the variation law of the recess pressure. When constant flow supply is used, the load carrying capacityis reduced by $42 \%$ at maximum tilt angle. Usingorifice restrictor, itis reduced by 33\%. There is no doubt that the best way to compensate is capillary restrictor, and itis reduced by $27 \%$ at maximum tilt angle.

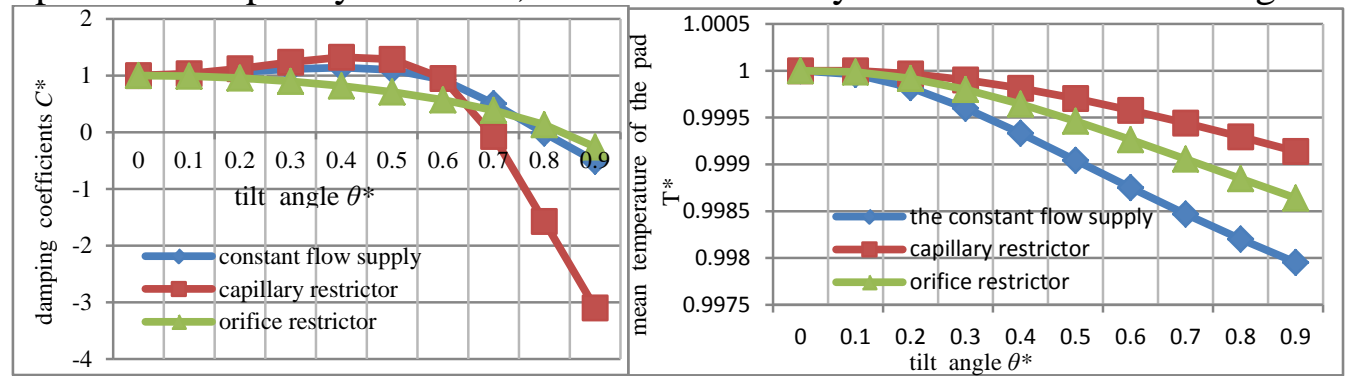

Fig.5 damping coefficients curvesFig.6 temperature of the pad curves 


\section{Damping coefficients}

The variation law of damping coefficients curves is shown in Fig.5. There is huge impact on damping coefficients because of tilt, and the damping coefficient even is negative when the tilt angle is larger.For the constant flow supply and capillary restrictor, the damping coefficients are consistent on the overall trend,which are slightly increased first and then a sharp decline in, but their biggest drop is different. When using capillary restrictor, it is maximallyreduced by $409.8 \%$. While using the constant flow supply, it is maximallyreduced by $151.2 \%$. When using orifice restrictor, the damping coefficient is smoothly decreased with the increase of tilt angle and maximallyby $126.1 \%$.

\section{Temperature of the pad}

The mean temperature of the pad will be decreased with the increasing of tilt angle, as shown in Fig. 6. Here, we can find that reduction is less than $0.2 \%$. And different restrictors also caninfluent it. While, when using the constant flow supply, the maximum amount of impact is about $0.2 \%$, and when using capillary restrictor,the number is about $0.1 \%$. There, temperature has a strong influence on performance of the pad, and the influence rate of thermal effects to load carrying capacitycurves are shown in Fig. 7. It can be calculated as $P_{w f}=100 \% *\left(w f_{\mathrm{n}}-w f_{\mathrm{t}}\right) / w f_{\mathrm{n}}$. While, $w f_{\mathrm{n}}$ isthe load carrying capacity without considering the influence of the pad, and $w f_{t}$ is the load carrying capacity considering the influence of the pad. Seen from the figure, the influence of temperature on the load carrying capacity is around $0.4 \%$ and increases with the increase of the tilt angle. Meanwhile, when using the constant flow supply, the influence is the largest, and it is the smallest when using capillary restrictor.

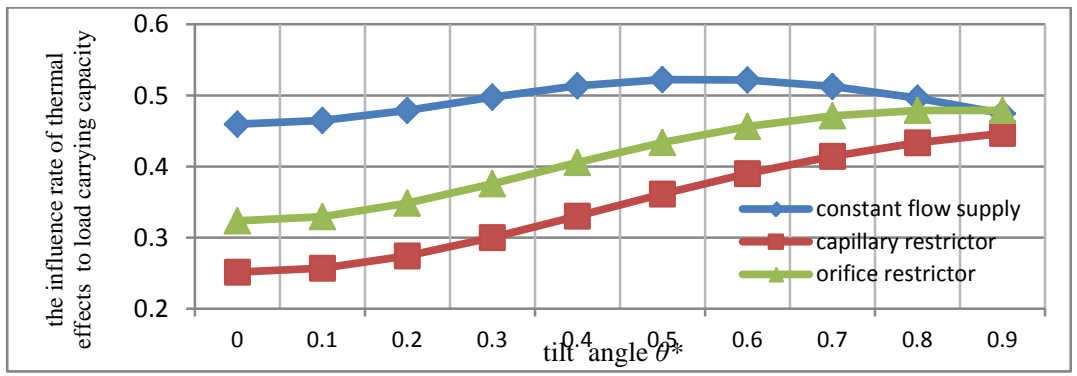

Fig.7 the influence rate of thermal effects load carrying capacitycurves

\section{Conclusion}

In this paper, energy equationandReynolds equationare established when the preloaded oil pad of the hydrostatic turntable tilts, and the influence of tilt on pad bearing properties is analyzed using different supply systems.The results show that the tilt has agreater impact on recess pressure, the load carrying capacityand damping. Thereinto, recess pressure and the load carrying capacity reduce by $10 \%$ to $50 \%$ at the maximum tilt angle,and the reduction in damping is even more than $100 \%$.Different methods of supply systems may also affect the anti-tilt abilityof the pad. In general, constant pressure supply system is better than constant flow supply system, and this is due to the constant pressure supply will increase the compensation effect when tilting to make itsanti-tilt ability improved.

\section{Acknowledgements}

The research work was supported byNational Science and Technology Major ProjectNo. 2013ZX04013-011 and Jing-Hua Talents Project of Beijing University of Technology

\section{References}

[1]Bassani R, Piccigallo B. Hydrostatic lubrication[M]. Elsevier, 1992.

[2]EskildStorteig, Maurice F White. Dynamic characteristics of hydrodynamically lubricated 
fixed-pad thrust bearings.Wear, 1999, 232(2):250-255.

[3]Guo Hong, Lai Xinmin, Cen Shaoqi. Theoretical and experimental study on dynamic coefficients and stability for a Hydrostatic/Hydrodynamic conical Bearing.Tribology,2009,131(4):1701-1707.

[4]Gao Dianrong, Zheng Dan, Zhang Zuochao. Theoretical Analysis and Numerical Simulation of the Static and Dynamic Characteristics of Hydrostatic Guides Based on Progressive Mengen Flow Controller.Chinese Journal of Mechanical Engineering, 2010, 23(6):709-716.

[5]Lin JR.Surface roughness effect on the dynamic stiffness and damping characteristics of compensated hydrostatic thrust bearings.International Journal of Machine Tools \&Manufacture, 2000,40:1671-1689.

[6]Ahmad W Yacout, Ashraf S Ismaeel, Sadek Z Kassab.The combined effects of the centripetal inertia and the surface roughness on the hydrostatic thrust spherical bearings performance.Tribology International,2007,40:522-532.

[7]NB Naduvinamani, BN Hanumagowda, SyedaTasneemFathima. Combined effects of MHD and surface roughness on couple-stress squeeze film lubrication between porous circular stepped plates. Tribology International.2012,56:19 29.

[8]Sharma S C, Yadav S K. Performance analysis of a fully textured hybrid circular thrust pad bearing system operating with non-Newtonian lubricant[J]. Tribology International, 2014, 77: 50-64.

[9]Ramesh A, Akram W, Mishra S P, etal. Friction characteristics of microtextured surfaces under mixed and hydrodynamic lubrication[J]. Tribology International, 2013, 57: 170-176.

[10]Bassani R. The thermohydrodynamic flow equations for hydrostatic thrust bearings[J]. Meccanica, 1970, 5(4): 262-269.

[11]Laraqi N, RashidiM, Garcia De Maria J M, etal. Analytical model for the thermo-hydrodynamic behaviour of a thin lubricant film[J]. Tribology International, 2011, 44(9): 1083-1086.

[12]Durany J, Pereira-Pérez J, Varas F. Some aspects of lubrication in heavy regimes: Thermal effects, stability and turbulence[J]. Mathematics and Computers in Simulation, 2014, 102: 90-103.

[13]Deresse G A, Sinha P. THD analysis for finite slider bearing with roughness: special reference to load generation in parallel sliders[J]. Actamechanica, 2011, 222(1-2): 1-15. 